\title{
Fatigue Life of Dental Implant- A Review
}

\author{
U.R.Patil $^{1}$, P.N.Dhatrak ${ }^{2}$, B.M.Shinde ${ }^{1}$ \\ ${ }^{1}$ (Department of Mechanical Engineering, JSPM'S RSSOER, Narhe, Pune-411041 India) \\ ${ }^{2}$ (Department of Mechanical Engineering, MAEER'S MIT, Pune - 411038 India)
}

\begin{abstract}
The reliability and stability of the implant abutment and implant-bone interfaces plays a great role for evaluation of the long-term success of a dental implant. In general, fatigue life depends on many factor such as implant itself, physical properties of bone as well as on other morphological characteristics that are patient dependent. This review studies the fatigue behaviour of dental implants made of commercially pure titanium alloy Ti-6Al-4V. The success or failure of an implant is determined by how the stresses at the bone-implant interface are transferred to the surrounding bones. Maximum stress concentration was presented in the connection of abutment-screw-implant as well as the first threads of the implant where the failure of implant occurs. Overload is the most common reason for implant failure. Source of the overload is due to patient parafunction habits and incorrect prosthesis design, which is responsible for creation of undesired bending moments. The prediction of fatigue life of the dental implant the finite element stress analysis with computer code of ANSYS was used. Fatigue life of dental implant is calculated based on Goodman, Soderberg, Gerber and Mean-Stress theories.
\end{abstract}

Keywords - Dental Implant, Fatigue Life, FEA.

\section{INTRODUCTION}

Dental implant are subjected to many loading cycles during mastication, which may cause them to break. When natural teeth are lost in cases such as deformities at birth, damage due to oral infection or accidental injuries at that time dental implant are used as replacement to natural teeth. Dental implant act as foundation to hold the artificial tooth into its proper position in human jaw. Implantation is done by surgical procedure which is very complicated, during implantation stresses are developed at implant-bone interface which directly related with the success of implantation and quick relief for patients.

Today, biomedical titanium alloy Ti-6Al-4V is used for the fabrication of dental implant. The reliability and stability of the implant abutment and implant-bone interfaces plays a great role for evaluation of the long-term success of a dental implant. In general, Fatigue life depends on many factor such as implant itself, physical properties of bone as well as on other morphological characteristics that are patient dependent.

The success or failure of an implant is determined by how the stresses at the bone-implant interface are transferred to the surrounding bones. The 3D finite element analysis (FEA) used for the evaluation of stresses on the implant and its surrounding bone. In the review, many researcher consider only static loading effect on the implant however dynamic loading and fatigue effects has not considered formally. Fatigue life of dental implant is calculated based on Goodman, Soderberg, Gerber and Mean-Stress theories.

\section{MATERIALS AND METHODS}

Titanium and titanium alloys are used materials for dental implant due to their good biocompatibility, excellent corrosion resistance and suitable mechanical properties. Loading of the implant, in 3-D with forces in lingual, an axial, and a mesiodistal direction as shown in Fig.1. These forces give masticatory force of $118.2 \mathrm{~N}$ in angle of approximately $75^{\circ}$ to the occlusal plane. The FEM model was fixed at the bottom surface of bone as shown in Fig 1. J.M.Ayllon, et al. [1] developed a methodology to estimate the life of an implant based on material properties and on a numerical model of the implant. He analysed the influence of different factors such as stress concentration at the external thread and the surface treatment. The stresses in the implant have been modelled with ANSYS, from these stresses the stress intensity is calculated and the initiation and propagation phases are analysed. He concluded that the surface treatment on implant gives a reduction in fatigue strength and high stress concentration at thread root where crack initiates. M.A.L.Hernandez, et al. [3] studied the failure examination included visual inspection, chemical analysis, metallography, microhardness testing, macroscopic and microscopic fractographic observations using scanning electron microscope with EDS (Energy-dispersive $\mathrm{X}$-ray spectroscopy). The fracture surface was studied by scanning electronic microscope (SEM) and energy 
disperse spectroscopy (EDS) to identify the failure mechanism. Horea T.Ilies, et al. [4] reported that the fatigue failure is estimated using the stress based approach, these approaches are based on stress amplitude compared to number of cycles (S-N) curves that have been established for a given material. He developed a finite element based fatigue model for dental implants, and correlate its results with analytical predictions and physical measurements.

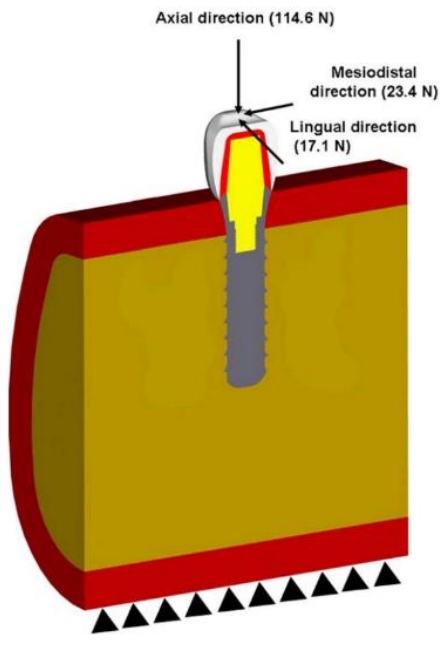

Fig 1. Load and boundary condition of FEM model [7]

M.A.Perez [5] was applied a probabilistic computational approach to predict the fatigue life of three different commercial dental implants. His technique was based on the combination of a probabilistic finite element method (PFEM) and a cumulative damage approach known as B-model. The random distribution of stresses/strains in the implant was computed by means of probabilistic finite element method. This stress distribution was directly related to the random properties of the occlusal loads acting on the implant. Oguz kayabas, et al. [7] investigated static, dynamic and fatigue behaviours of dental implant. In his study, static, dynamic and fatigue behaviours of dental implant are investigated. For the loading conditions tested, the maximum stress values are lower than the yield strength of abutment and prosthetic screws of the implant/abutment joint systems. It shows that the implant is durable in static and dynamic loading condition. M. Jamshidinia et al. [8] studied Fatigue properties of a dental abutment with a lattice structure. He used Electron Beam Melting (EBM) to produce the dental abutments, made of Ti-6Al-4 V. Four different levels of cyclic loads such as $100 \mathrm{~N}, 200 \mathrm{~N}, 300 \mathrm{~N}$, and $500 \mathrm{~N}$ were applied by using a sinusoidal wave form, and the loading ratio of $10 \%$. He suggested that the fatigue crack can be originated from the partially sintered powder particles. Finally, a regression equation was established from the experimental results that can be used to predict the fatigue life of the dental implant. Benaissa ali et al [11] reported that mechanical behaviour of a dental prosthesis under two loading types (dynamic and dynamic within overload). He concluded that the mechanical stress is propagating in the areas of bones which are closer to the dental implant, and reduction in magnitude towards the outer region. The mechanical stress reaches the highest in areas of implant-bone interface which are immediately next to the neck of the dental implant, the maximum Von Mises stress occurs inside the dental implant itself. Krren Shemtov [12] studied three groups of implants with different diameters $(3.3 \mathrm{~mm}, 3.75 \mathrm{~mm}$ and $5 \mathrm{~mm})$, were tested under static as well as a cyclic compressive loading. In the cyclic test, the machine stopped operating when the structure failed or when it reached $5 * 10^{\wedge} 6$ cycles without failure. A macroscopic failure analysis was performed, which evaluated and located the failure modes obtained, followed by a microscopic failure analysis with the help of scanning electron microscopy (SEM). Ferdinan do felli, et al. [13]in his study two different titanium dental implants are analysed in order to evaluate their mechanical strength. Implants are tested under fatigue loading in different ways. The first implant is subjected to tensile stress while the second one is dynamically loaded with a complex stress system which is similar to the one that takes place in the actual mastication action. The fatigue tests performed on the two dental implants using a hydraulic-drive fatigue test machine, as well as fracture surface analyses which has to be carried out by a scanning electron microscope (SEM). J. Fail Anal, et al. [16] performed a failure analysis on a titanium-based dental implant and connect the possible reasons of failure with the associated material requirements. Evaluation techniques included metallographic analysis by using optical microscopy and fractographic analysis by using scanning electron microscopy (SEM). The results of the examinations suggested that, in spite of their adequate microstructures, the implants fractured due to the overload generated by stress raisers which were found in the implants. Min Gun kim [17] evaluate the fatigue limit under normal conditions and in a simulated body environment. This

$5^{\text {th }}$ National Conference RDME 2016, 10-1 $1^{\text {th }}$ March 2016.2 2|Page


study used the GS II model of Osstem Implant as the implant specimen. The fixture was the internal type made of titanium, measuring $4 \mathrm{~mm}(\varnothing)$ by $11.5 \mathrm{~mm}(\mathrm{~L})$. The abutment was the hex standard type, at $5 \mathrm{~mm}(\varnothing)$ and 2 $\mathrm{mm}$ (L) (Gingival/Height). The base material of this abutment screw consists of Ti-6Al-4V, and its surface is coated with tungsten carbide at a depth of $2 \mu \mathrm{m}$ to $3 \mu \mathrm{m}$. A hemispherical loading member on which the load was applied during the test was prepared in accordance with the ISO 14801 specification. Song park et al [18] studied the fatigue limits for five implant-abutment combinations. Each implant-abutment system was tightened with a torque of $32 \mathrm{~N}-\mathrm{cm}$. The test specimen was loaded at an inclination of 30 degrees with respect to the loading axis which is $11 \mathrm{~mm}$ away from the fixed point. A cyclic compression load was applied at loading cycles of $10 \mathrm{~Hz}$ using a hydraulic dynamic testing machine. In his study, three types of implants from among those in the AVANA implant system were selected as the experimental materials.

\section{FINITE ELEMENT ANALYSIS}

J.M. Ayllon, et al. [1] in his study the geometry of the implant was defined with the software SOLIDWORKS and then exported to ANSYS where implant was meshed and solved. The purpose of this numerical model is to obtain the stresses and strains in the implant, as well as the Stress intensity factor along the path of the crack. The model of the implant required the use of a total of 3,11,064 solid 187 10-node tetrahedral elements. M.A.L.Hernandez, et al. [3] performed finite element analysis (FEA) using the software ANSYS Workbench 14.0 in order to find out the stress distribution of the system. The mechanical simulation was carried out at static mode for two loads acting on the implant at cantilever with an angle of $30^{\circ}$. The meshed model contains total 31,865 tetrahedral elements and 58,642 nodes. Horea T. Ilies, et al. [4] obtained a digitized image of the implant, which was then converted into a binary image in Matlab. Boundary points of the implant were created from the binary image and were used to construct the solid model of the implant. These dimensions were then used to create a faithful solid model of the implant in a commercial solid modeller. The maximum Von Mises stress was computed for each element in ABAQUS. The FEA analysis results were used to produce S-N curves using the four different methods. M.A.Perez [5] in his study the implants were scanned in a MicroCT (GE Locus, GE Healthcare, London, ) to obtain their geometry. Then, using computerized tomography (CT) data, the second premolar region of a human mandible was created using Mimics (Materialise, Leuven, Belgium). Semi-automatic segmentation was used for the region of the mandible to get difference between cortical and trabecular bone. Stereo lithographic files were exported from Mimics to an automatic mesher (Harpoon Sharc Ltd, Manchester, UK) where the FE meshes were generated for the different models. The number of tetrahedral element and nodes used in this study for all the models were approximately 7, 00,000 and 4, 00,000 respectively. All the analyses were performed with the commercial FE software package Abaqus v.6.9. Oguz Kayabas, et al. [7] in FEA the implant and its superstructure were modelled using CAD software Pro/Engineer 2001. The FEM model consists of total 2,98,070 four-node tetrahedron elements; 44,127 elements for implant, 13,534 elements for abutment, 4,987 elements for metal framework, 9,902 element for feldsphatic porcelain, 60,762 element for bone, 19,658 element for gingiva. Elements in implant, abutment, metal framework, feldsphatic porcelain, bone and gingiva correspond to SOLID45 type tetrahedron elements in ANSYS with each node having three degree of freedom.

\section{RESULT AND DISCUSSION}

1. The total life of implants for short life implants does not change due to surface treatment. Only for long life implants the surface treatment will have an effect on the initiation life and total life. The Initiation life that is always below $5 \%$ of total life.

2. Implant failure occurs at the region where there is drastic change in geometry such as the neck region of implant. The maximum concentration of stresses were found in the connection of abutment-screwimplant.

3. Autoclaving process can significantly decrease the fatigue life of dental implant. The autoclaved implants failed much sooner than the original implants that had not been subjected to thermal gradient.

4. The highest failure probability was obtained at the upper screw-threads, due to high stress concentrations appeared around these regions. Maximum stresses were obtained at first thread of the implant for both static and dynamic loading conditions.

\section{CONCLUSION}

Considering the results of this review, the following conclusions can be derived as:

1. Implant design parameters such as pitch, diameter and length is important to ensure long-term fatigue performance for dental implants.

2. The combination of sharp notches (thread) and narrow metal cross section might be harmful for fatigue resistance. 
[22] F. J. Gil, M. Herrero-Climent, and P. La'zaro J. V. Rios "Implant-abutment connections: influence of the design on the microgap and their fatigue and fracture behavior of dental implants." J Mater Sci: Mater Med (2014) 25:1825-1830

[23] C. Barbosa, J. L. do Nascimento, I. M. V. Caminha, I. C. Abud "Premature Failure in Orthopedic Implants: Analysis of Three Different Cases." J Fail. Anal. And Preven. (2009) 9:67-73 